\title{
Two Cases of Submandibular Sialolithiasis Detected by Cone Beam Computed Tomography
}

\author{
Melek Tassoker, Sevgi Ozcan \\ Department of Oral and Maxillofacial Radiology, Necmettin Erbakan University, Konya, Turkey
}

\begin{abstract}
Salivary gland stones can be detected with different diagnostic methods. Plain radiography, sialography, computed tomography (CT), cone-beam CT (CBCT), ultrasonography (US), magnetic resonance imaging (MRI), and nuclear scintigraphy / positron emission tomography (PET) all play roles in the diagnosis of salivary gland lesion. The aim of this report was to present clinical and radiological features of two submandibular sialoliths detected by CBCT and to discuss the clinical value of CBCT in diagnosis of submandibular gland sialolith.
\end{abstract}

Keywords: sialolith, cone beam CT, submandibular gland

\section{INTRODUCTION}

Sialolithiasis is a disease characterized by the occurrence of sialoliths in the different parts of the salivary glands [1]. It is the most common disease of the salivary glands and is a major cause of salivary gland dysfunction [2,3]. It is estimated that it affects 12 in 1000 of the adult population [2,4] and usually appears between the age of 30 and 60 years [4]. Males are affected twice as often as females, children are rarely affected [5].

More than $80 \%$ of sialoliths occur in the submandibular gland or its duct, $4-10 \%$ occur in the parotid gland, and $1-7 \%$ occur in the sublingual gland or minor salivary glands [5,6]. Sialolith formation is more likely to occur in the submandibular gland due to its anatomic position requiring the salivary flow against gravity, longer and more tortuous duct and production of alkaline saliva rich in mucin. Most submandibular calculi are detected as radiopaque formations in plain radiographs and as radiolucent filling defects in sialography. Approximately $20 \%$ of sialoliths are not radiopaque, and sialography or sialendoscopy may be required to diagnose them. Salivary calculi are usually unilateral and do not cause dry mouth [7].

Sialoliths are hard formations, with round or oval shape and a variety of sizes. Their color ranges from white to brown and have nodular surface with prominences, the smaller ones looking like coral. Sialoliths are usually composed of a round organic core, intensely calcified, that is surrounded by alternative layers of organic and inorganic substances. The organic layers are composed of condensed mucus, mucopolysaccharides, glucoproteins, cellular elements and lipids, while the inorganic material is composed of calcium phosphate, calcium carbonate and trace elements [8].

Microcalcifications occur frequently in the submandibular glands [1]. In a study of normal submandibular glands at autopsy Scott [9] found that solid-appearing calcified and noncalcified micro deposits in small ducts increased with age. Seifert et al. [10] stated that the earliest changes of sialolithiasis result from electrolyte imbalance in the saliva and the filling and distension of small ducts with viscous mucoid secretions. Lamellar microcalcifications would form later by precipitation of additional secretions and appositional growth.

Usually sialoliths measure from $1 \mathrm{~mm}$ to less than $1 \mathrm{~cm}$. They rarely measure more than 1.5 $\mathrm{cm}$. In the literature, giant sialoliths are classified as those exceeding $15 \mathrm{~mm}$ in any one dimension or more than $1 \mathrm{gm}$ in weight [11]. It is believed that a calculus may enlarge at the rate of approximately 1- $1.5 \mathrm{~mm}$ per year [12]. The growing stone causes an increasing obstruction of salivary secretion, which leads to swelling, pain, and infection of the gland and eventually medical intervention. However, if the duct adjacent to the sialolith is able to dilate, allowing nearly normal secretion of the saliva around the stone, it might be asymptomatic for a long period and eventually a giant calculus will be created. As the stone increases in size beyond the ability of the duct to dilate, a sialo- oral fistula will develop and the sialolith will partially protrude into the oral cavity [11].

Sialolithiasis is easy to diagnose on the basis of its clinical features [4]. Bimanual palpation of the floor of the mouth, in a posterior to anterior direction, reveals a palpable stone in a large number of cases of submandibular calculi formation [6]. Painful swelling, swelling of the involved gland during eating, digitally expressible pus, and visual or tactile detection of calculi within major salivary gland duct orifices are typically associated clinical symptoms [5,13]. 
Sialolithiasis can be managed by surgical or nonsurgical therapeutic approaches [3]. Most stones will respond to antibiotics, combined with simple sialolithotomy [4]. The primary objective of treatment for giant sialoliths is the restoration of normal salivary secretion. The treatment options for submandibular sialoliths are intraoral removal of stone, interventional sialendescopy, resection of the gland [3] and extracorporeal shock wave lithotripsy (ESWL) [2]. The appropriate treatment method is chosen according to the size, location and number of sialoliths. Small sialoliths can be removed through the duct orifice using bimanual palpation. Larger or proximally located sialoliths in the duct require surgical removal through oral cavity. Submandibular gland resection is indicated when a sialolith with a substantial mass (12 $\mathrm{mm}$ or more) is located within the gland and intraoral surgical access is not possible [3]. ESWL is an effective, noninvasive alternative approach in the surgical removal of salivary stones, reserving surgery for recurrent or complicating salivary lithiasis. The principle of ESWL is to reduce the size of calculi to small fragments, the diameter of which does not block the flow of saliva and can be washed away by natural flow [2]. Andertta et al. [14] discussed the limitations of ESWL in the removal of stone with a diameter of $>10 \mathrm{~mm}$, as it is difficult to reduce the fragments and also in cases of acute inflammation. If the stone can be palpated, it is best to remove it transorally [12].

Radiographic examination is essential in diagnosing the lesions, and useful for planning further management, whether surgery or alternatives $[15,16]$. Plain radiography, sialography, computed tomography (CT), cone-beam CT (CBCT), ultrasonography (US), magnetic resonance imaging (MRI), and nuclear scintigraphy / positron emission tomography (PET) all play roles in the diagnosis of salivary gland lesions [17].

Two cases of sialolithiasis with varying presentations and clinical features reported to our Department of Oral and Maxillofacial Radiology. A brief discussion based on the clinical and radiological examination of sialolithiasis is being reported.

\section{Case 1:}

\section{CASE REPORT}

A 48-year-old female patient came with a complaint of pain in the maxillar anterior implant site. Additionally, there was history of increase of the pain and swelling during mealtimes on the left side of mandible for approximately two years. Medical history of the patient was not remarkable. Clinical examination revealed neither palpable mass nor swelling on the floor of the mouth (Figure 1). Salivary secretion seemed normal (Figure 2).

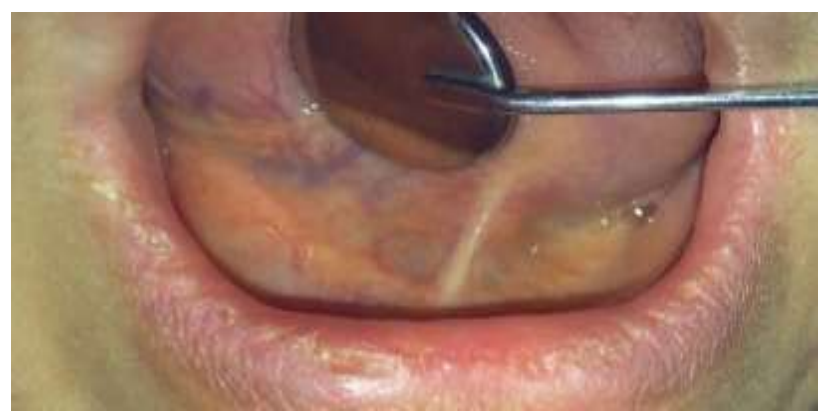

Figure 1. Intraoral image showing the floor of the mouth

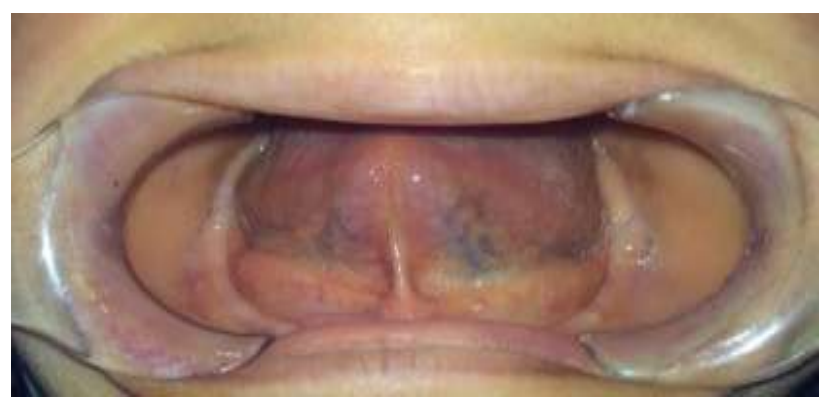

Figure 2. Patient's salivary secretion seemed normal

Panoramic image was obtained for initial radiologic examination. Panoramic image revealed an osteosclerotic area on the left corpus of mandible (black arrow) and a round radioopaque mass superimposed on the left angle of mandible (blue arrow) (Figure 3).

DOI: $10.9790 / 0853-150810124129 \quad$ www.iosrjournals.org $\quad 125 \mid$ Page




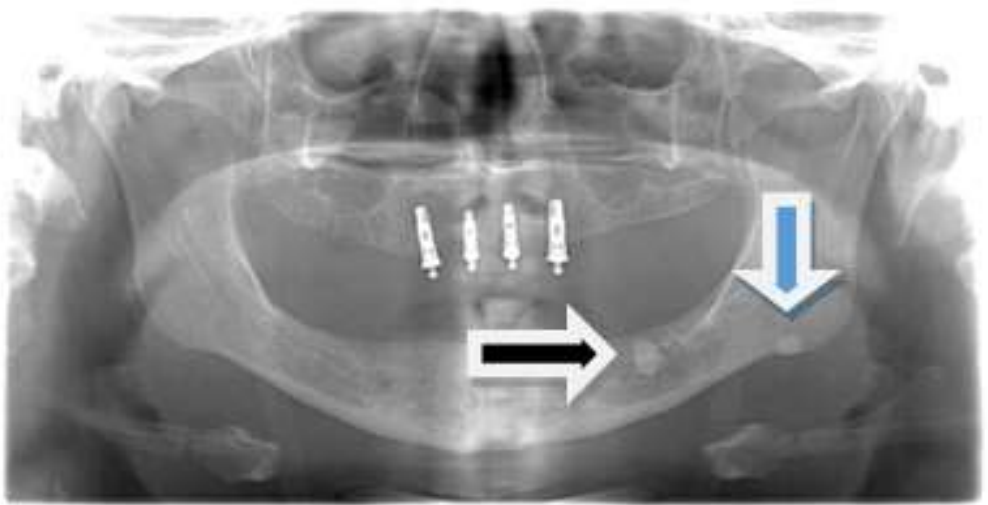

Figure 3. Panoramic radiograph of the patient

There was no reason detected in clinical and panoramic radiographic examination of the paitent with regarding the pain of maxillar anterior implant side. CBCT scan (J. Morita, 3D Accuitomo 170, MFG corp, Kyoto, Japan) confirmed the presence of the submandibular sialolith $(5.15 \times 5,13 \times 2,8 \mathrm{~mm}$ size) (Figure 4) and also demonstrated maxillar anterior implant penetration to the maxillar nasopalatine canal (Figure 5). Patient was posted for the surgical removal of implants and submandibular sialolith.

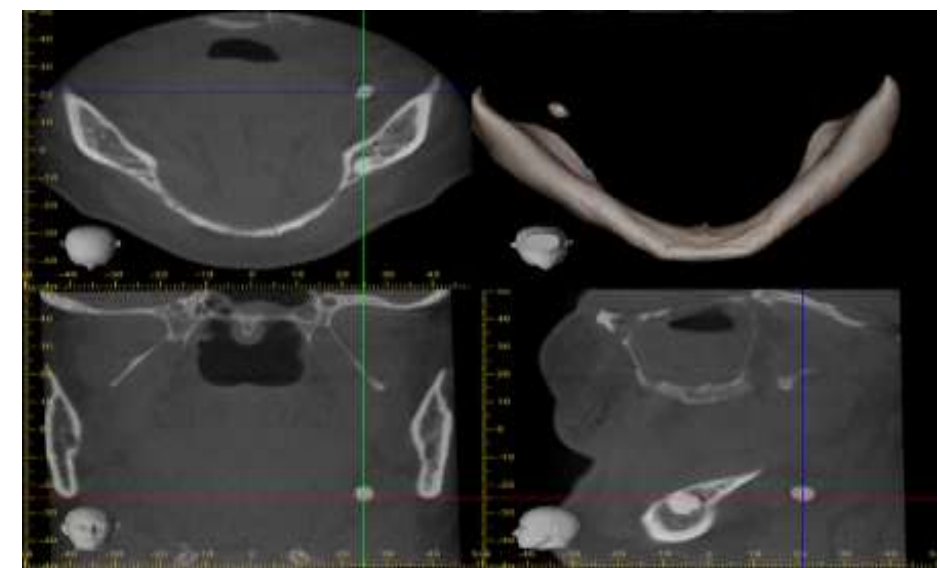

Figure 4. Axial, coronal, sagittal and 3D CBCT reconstructions showing the sialolith

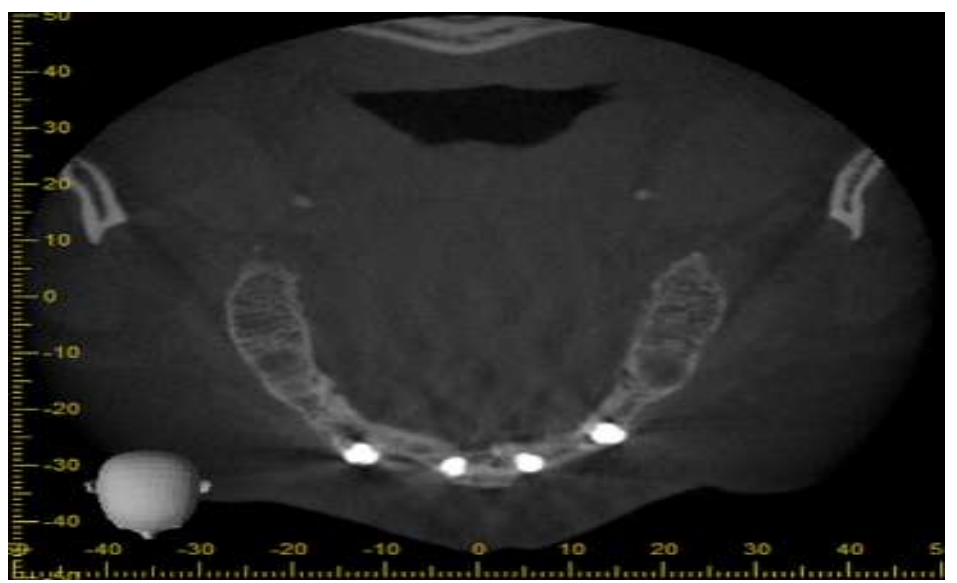

Figure 5. Axial view showing the nasopalatine canal penetration of the implant

Case 2

A 64-year-old female patient was referred to our clinic for tooth loss and prosthetic requirement. Her medical history included hypertension and hyperthyroidism. Ortopantomography was performed for initial radiologic examination and it showed a radioopaque mass on the inferior border of left mandible (arrow) (Figure 6). 


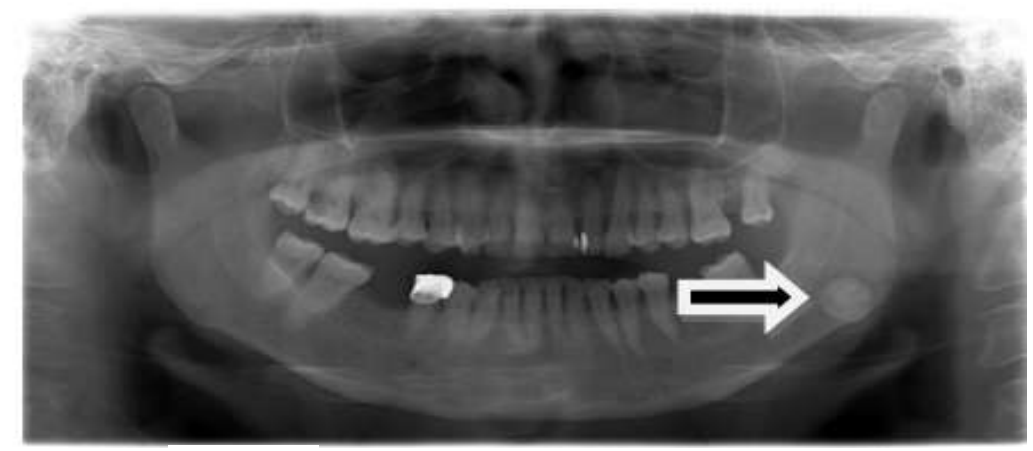

Figure 6. Panoramic radiograph of the patient

Intraoral bimanual examination of the related area revealed a swelling localized in the left side of the floor of mouth, solid to touch and not adherent to any deeper structure. Salivary secretion seemed normal. The diagnosis of salivary calculus of the Wharton's duct was confirmed by CBCT scan (with $90 \mathrm{kVP}, 5 \mathrm{~mA}, 17,5$ seconds irradiation time) that showed a radiopaque mineralized amorphous formation about $11,62 \times 12,32 \times 8,20 \mathrm{~mm}$ large within the submandibular gland (Figure 7). Patient was referred to Department of Oral and Maxillofacial Surgery for the surgical removal.

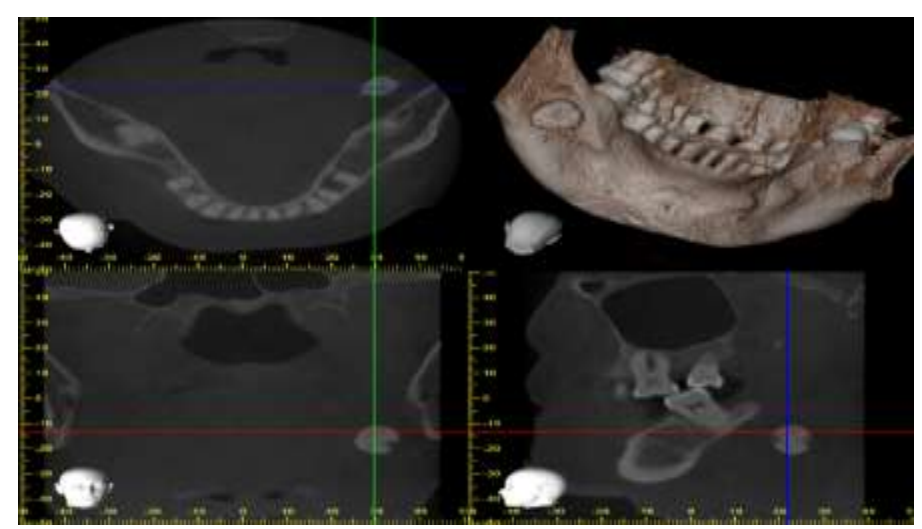

Figure 7. Axial, coronal, sagittal and 3D CBCT reconstructions showing the sialolith

\section{DISCUSSION}

The sialolithiasis is a very common disease, which can be asymptomatic for a long time [1]. The symptoms begin whenever the lumen of Wharton's duct is obstructed by a salivary calculus with resultant accumulation of saliva causing pain and swelling of the involved gland. The stasis of saliva can lead to bacterial ascent into the parenchyma of the gland and therefore recurrent infections. Longterm obstruction of salivary flow and recurrent infections can lead to atrophy of the gland with resultant loss of secretory function and ultimately fibrosis [3].

The algorithm for imaging the salivary glands depends on the clinical scenario with which the patient presents to the clinician. Because the causes of the symptoms can be complex, identifying small calculi in the gland or salivary duct is important in order to identify the real cause [5].

Submandibular sialolithiasis can be visualized by different techniques as we mentioned in introduction. In our cases, in addition to panoramic radiographs, we used CBCT for diagnosis of sialoliths and identify their location. Compared with conventional techniques, and according to our current findings, CBCT was highly sensitive in showing the location and size of sialoliths.

Conventional 2D radiography is failure-susceptible for small or less-calcified calculi especially if superimposed by well-calcified bone, and $10 \%$ to $30 \%$ of salivary stones are categorized as radiolucent [13]. If a small calculi are present or calculi are superimposed on anatomical structures, a second radiological 2D plane must be performed [13]. Panoramic images could be both distorted and magnified, which means that unreliable results could be produced [5]. Occlusal radiographs are useful for the diagnosis of radioopaque submandibular sialolithiasis [18] however evaluation of the salivary glands by plain radiography is of limited clinical value [17].

Sialography has up to $100 \%$ diagnostic accuracy [5] and creates excellent contrast resolution and allows small stones or strictures to be detected [16]. However its use is contraindicated in patients who are having contrast allergy [4], acute infections and orifice stones [5]. The technique requires 
injecting contrast media into the Stensen's or Wharton's duct of the major salivary glands to identify the outline of the ductal anatomy and any presence of sialoliths [16]. Despite several limitations of sialography, it remains widely used for the diagnosis of salivary gland diseases, which affect the ductal system; in addition it provides the ability to assess the suitability for interventional procedures and radiolucent stones [17]. CBCT [5], CT and US [17] have shown to be of limited value in visualizing the ductal system. CBCT offers improvement in demonstration of the ductal system over conventional sialography [19]. Conventional sialography uses panoramic, occlusal, and postroanterior radiographs, which provide appropriate views for examining the major salivary glands [17].

Sialoendoscopic system was developed in the 1990's as an endoscopic technique, and provides to examine the ductal system completely due to the scopes are so small. Sialoendoscopy can be used for both diagnostic and treatment purposes [6]. It is mainly used in the treatment of inflammatory condition of the salivary glands and obstruction of the salivary ducts [2]. It is a technique for treating obstructions of the ductal system and can be used with operation in large salivary stones [6]. Yu et al. [20] reported that sialoendoscopy was superior to plain film or even to magnetic resonance imaging for especially the small and more distal located stones. In this report, as sialolith were observed clearly in CBCT, no further investigations were performed for diagnosis.

By applying high-resolution imaging protocols, 3D medical CT can successfully display even the smallest or semicalcified calculi, but at the expense of high radiation doses to patients [13].

MRI is reported to be a valuable additional diagnostic method in difficult cases, or when further soft tissue diagnosis is necessary. It may depict the status of the gland parenchyma affected by sialolithiasis. That is to say, it may reflect the chronic and acute nature of the obstruction [5]. MRI cannot be considered a routine diagnostic tool for salivary calculus diagnosis because of the laborious technical effort and expense per investigation as well as its relatively low level of availability [13]. MR sialography could replace the conventional sialography in depicting the dilated duct and also sialolith in it [5].

US is the least invasive method [13] and be used in patients with acute sialadenitis [5]. Failure has been reported in cases of small semicalcified stones [13].

Despite the advantages of 3D medical CT or MRI evaluations, US and 2D radiography are routinely used owing to cost-effectiveness, availability, and lower radiation dosages. Since the introduction of CBCT to dentistry in 1998, its application has become routine for presurgical, dentalimplant, and third-molar assessments [13]. CBCT is as reliable as medical CT in making accurate measurements of anatomical and pathological structures [13]. The main limitation of CBCT in diagnosing sialolithiasis is the lack of a soft tissue window compared to medical CT and medical CT is often the best initial study for the evaluation of a painful gland, including the soft tissue [5].

\section{CONCLUSION}

Because of its high diagnostic information-to-radiation-dose ratio, $\mathrm{CBCT}$ is the preferable imaging modality for salivary calculus diagnosis. It provides useful information for the measurement and location of submandibular sialolithiasis. However it cannot provide additional information about ductal anatomy and relationship between the sialolith and surrounding soft tissue. Therefore, other advanced forms of imaging, such as medical CT and sialography techniques may be effective.

\section{REFERENCES}

[1]. Batzakakis D, Apostolopoulos K, Bardanis I. A case report of coexistence of a sialolith and an adenoid cystic carcinoma in the submandibular gland. Med Oral Patol Oral Cir Bucal 2006; 11(3):e286-8.

[2]. Bhullar RS, Dhawan A, Bhullar K, Malhota S. Giant submandibular gland duct sialolith mimicking an impacted canine tooth. Natl J Maxillofac Surg 2015; 6(1):89-92.

[3]. Arslan S, Vuralkan E, Çobanoğlu B, Arslan A, Ural A. Giant sialolith of submandibular gland: report of a case. Journal of Surgical Case Reports 2015; (4):1-3.

[4]. Kuruvila VE, Bilahari N, Kumari B, James B. Submandibular sialolithiasis: Report of six cases. J Pharm Bioallied Sci 2013; 5(3):240-242.

[5]. Miloğlu Ö, Çağlayan F, Ezmeci T, Dağıstan S, Demirtaş Ö. Multiple cases of submandibular sialolithiasis detected by cone beam computed tomography. J Dent Fac Atatürk Uni 2010; 3(20):189-193.

[6]. Alkurt MT, Peker I. Unusually large submandibular sialoliths: Report of two cases. Eur J Dent 2009; 3(2):135-139.

[7]. Austin T, Davis J, Chan T. Sialolithiasis of submandibular gland. J Emerg Med 2004; 26(2):221-3.

[8]. Harill JA, King JS Jr, Boyce WH. Structure and composition of salivary calculi. Laryngoscope 1959; 69(5):481-92.

[9]. Scott J. The prevalence of consolidated salivary deposits in the small ducts of human submandibular glands. J Oral Pathol 1978; 7(1):28-37.

[10]. Seifert G, Miehlke A, Haubrich J, Chilla R. Diseases of the salivary glands: Pathology, diagnosis, treatment, facial nerve surgery. Stuttgart, George Thieme Verlag KG 1986.

[11]. Bodner L. Giant salivary gland calculi: Diagnostic imaging and surgical management. Oral Surg Oral Med Oral Pathol Oral Radiol Endod 2002; 94(3):320- 3 .

[12]. Iqbal A, Gupta AK, Natu SS, Gupta AK. Unusually large sialolith of Wharton's duct. Ann Maxillofac Surg 2012; 2 (1):70- 3. 
[13]. Dreiseidler T et al. Salivary calculus diagnosis with 3-dimensional cone-beam computed tomography. Oral Surg Oral Med Oral Pathol Oral Radiol Endod 2010; 110(1):94-100.

[14]. Andretta M, Tregnaghi A, Prosenikliev V, Staffieri A. Current opinions in sialolithiasis diagnosis and treatment. Acta Otorhinolaryngol Ital 2005; 25(3):145- 9.

[15]. Jäger L, Menauer F, Holzknecht N, Scholz V, Grevers G, Reiser M. Sialolithiasis: MR sialography of the submandibular duct-an alternative to conventional sialography and US? Radiology 2000; 216(3):665-71.

[16]. Burke CJ, Thomas RH, Howlett D. Imaging the major salivary glands. Br J Oral Maxillofac Surg 2011; 49(4):261-9.

[17]. Waseem Z, Forte V. An unusual case of bilateral submandibular sialolithiasis in a young female patient. Int J Pediatr Otorhinolaryngol 2005; 69(5):691-4.

[18]. Abdel-Wahed N, Amer ME, Abo-Taleb NSM. Assesment of the role of cone beam computed sialography in diagnosing salivary gland lesions. Imaging Sci Dent 2013; 43(1):17-23.

[19]. Li B, Long X, Cheng Y, Wang S. Cone beam CT sialography of Stafne bone cavity. Dentomaxillofac Radiol 2011; 40(8):519-23.

[20]. Yu CQ, Yang C, Zheng LY, Wu DM, Zhang J, Yun B. Selective management of obstructive submandibular sialadenitis. Br J Oral Maxillofac Surg 2008; 46(1):46-9. 